\title{
PREVENZIONE E PERCEZIONE DI FENOMENI CORRUTTIVI: ISTANZE DI DIFESA SOCIALE E CRISI DEL GARANTISMO PROCESSUALE PENALE
}

\author{
Gaspare Dalia* \\ "University of Salerno (Italy), Department of Legal Sciences (School of Law) \\ (gadalia@unisa.it)
}

\begin{abstract}
Constitutions and the International Charters of Rights are concerned to ensure the judicial verification of the unlawfulness of facts in compliance with the inalienable rights of the person and the presumption of innocence. Today, modern criminal procedural law, which has always been conditioned by different legal traditions, by different political-constitutional approaches and by the current "criminal emergencies", suffers further stress resulting from political and media communication on topics such as organized crime, terrorism and corruption, thus diverting the analysis from objective data and basing the discussion on questionable and instrumental evaluations. In this context, the demands of justice do not coincide with those of legality, but with those of social defense. In Italy, the disproportionate increase in anti-corruption measures has profoundly changed the procedural means of their assessment, with a clear danger for the rights of suspects and/or accused persons and with a consequent dangerous inquisitorial drift.

At the international level, however, new and better measures are being tested. The long-term goal is to identify objective criteria in order to measure corruption in each country, using real-time data. Only objective indicators will make it possible to conduct investigations that "measure" the corruption in countries in order to initiate more incisive actions to combat it.
\end{abstract}

Keywords: Corruption, Corruption Perceptions Index, Precautionary Measures, Prevention, Public Opinion, Repression, Social Defence, Transparency International. 


\title{
PARANDALIMI DHE PERCEPTIMI I DUKURIVE KORRUPTIVE INSTANCAT E MBROJTJES SOCIALE DHE KRIZAT E GARANTIZMIT PROCESUAL PENAL
}

\author{
Gaspare Dalia* \\ "Universiteti i Salernos (Itali), Departamenti i Shkencave Ligjore (Shkolla e së Drejtës) \\ (gadalia@unisa.it)
}

\begin{abstract}
Abstrakt
Kushtetutat dhe Kartat Ndërkombëtare të të Drejtave shqetësohen për sigurimin e verifikimit gjyqësor të paligjshmërisë së fakteve në përputhje me të drejtat e patjetërsueshme të personit dhe supozimit të pafajësisë. Sot, e drejta moderne procesuale penale, e cila ka qenë gjithmonë e ndikuar nga tradita të ndryshme juridike me qasje të ndryshme politiko-kushtetuese dhe nga "emergjencat penale" aktuale, vuan nga një stres $i$ shtuar që vjen nga komunikimi mediatik dhe politik mbi tema si krimi i organizuar, terrorizmi dhe korrupsioni, që e shkëpusin analizën nga të dhënat objektive dhe e bazojnë diskutimin në vlerësime të diskutueshme dhe instrumentale. Në këtë kuadër, kërkesat për drejtësi nuk përkojnë me atë të ligjshmërisë, por me ato të mbrojtjes sociale. Në Itali, rritja jo-proporcionale e masave kundër korrupsionit ka ndryshuar thelbësisht mënyrat proceduriale të vlerësimit të tyre me një rrezik të qartë për të drejtat e të dyshuarve dhelose personave të akuzuar dhe me një prirje të rrezikshme inkuizitore konsequente.

Megjithatë, në nivel ndërkombëtar, po testohen masa të reja dhe më të mira. Qëllimi afatgjatë është të identifikohen kriteret objektive për matjen e korrupsionit në çdo vend, duke përdorur të dhëna në kohë reale. Vetëm treguesit objektivë mund të bëjnë të mundur kryerjen e hetimeve që "masin" korrupsionin në vend në mënyrë $q \ddot{e}$ të nxisin veprime më të mprehta në luftën kundër tij.
\end{abstract}

Fjalë kyçe: korrupsioni, indeksi $i$ perceptimeve të korrupsionit, masat parandaluese, opinion publik, represioni, mbrojtja sociale, Transparency International

\section{I valori del diritto processuale penale}

Il sistema processuale penale ha sempre cercato il giusto punto di equilibrio tra l'ansia della riaffermazione del diritto sostanziale violato e le garanzie dell'imputato. È, questa, una realtà dalla quale non si può prescindere se si vuole cogliere il significato che al processo hanno riconosciuto le esperienze del passato.

Nelle direttive della legge delega, la risistemazione della disciplina del processo penale avvenuta in Italia nel 1988 tendeva a realizzare tanto i principi costituzionali, quanto i caratteri dell'accusatorietà dell'accertamento: i valori, che la dimensione costituzionale del processo esprime, esaltano il senso del riferimento; il richiamo ai principi 
costituzionali e al metodo non attesta una duplicità di scelta, ma unicamente la diversità di prospettiva nella quale ci si pone quando si invocano gli uni e, nel contempo, si richiamano i caratteri degli altri.

Storicamente, la metodologia del processo penale - perché qui proprio di metodo si tratta - ha oscillato tra i due massimi sistemi dell'accusatorio e dell'inquisitorio, riflettenti, in definitiva, i due opposti modi d'intendere il rapporto Stato-individuo nell'accertamento penale e nella gestione del bene della libertà personale: ma, la storia del processo penale non è tanto nella supremazia dell'un sistema sull'altro, quanto nella varietà dei tentativi intesi a combinarne i caratteri.

La differenza tra i due modelli è evidente: nel primo caso, l'accusa è già delineata nei suoi elementi costitutivi e, quindi, già esiste un'opinio delicti che legittima la contestazione di un fatto nel quale sono ravvisati gli estremi della trasgressione al precetto penale. Inquisito è, al contrario, colui nei cui confronti non si è evidenziata ancora un'accusa, ma si svolgono indagini tendenti proprio alla formulazione di questa.

Dal punto di vista logico, l'inquisizione non può non precedere l'imputazione perché il metodo inquisitorio caratterizza - e non può non caratterizzare - le attività investigative necessarie e sufficienti per formulare l'accusa, presupposto indefettibile per l'avvio dell'accertamento in chiave accusatoria.

L'attività inquisitoria è, in altri termini, necessariamente un'attività preprocessuale, che dà vita al processo se ed in quanto, esauritasi, consenta la formulazione dell'accusa.

Una volta che questa si sia materializzata nelle sue componenti essenziali, non dovrebbe più parlarsi di maggiore o minore inquisitorietà dell'accertamento, perché dovrebbero naturalmente espandersi le garanzie che si accompagnano alla formulazione dell'imputazione-accusa.

Se il soggetto "accusato" non può non essere quello precedentemente inquisito, non è prospettabile alcuna combinazione dei caratteri dell'uno e dell'altro metodo: quello accusatorio s'inserisce e nasce laddove termina l'inquisitorio; né può assolutamente vedere i suoi principi alterati per la commistione con l'altro.

Anche a voler tralasciare ogni ulteriore considerazione sul significato che storicamente ha assunto il modello inquisitorio, non si può non esser convinti dell'impossibilità di leggere nei metodi che la tradizione giuridica ci ha tramandato commistioni, più o meno consistenti, dei caratteri dell'una e dell'altra metodologia.

Una volta ritrovata la radice della funzione giurisdizionale nei principi costituzionali, non si può non riconoscere che tra processo e giurisdizione esiste una relazione di identità: se il processo attua la giurisdizione, questa non può non rivolgersi nel dicere ius del giudice secondo le forme e le prerogative che il processo contraddistingue.

In realtà, il processo è giurisdizione e la giurisdizione non è immaginabile senza un processo che in esso attui le garanzie della persona.

\section{La giurisdizione penale come strumento di difesa sociale}

La riflessione sui diversi punti di vista dai quali la dottrina ha esaminato il tema della giurisdizione penale pone costantemente in evidenza - quale che sia l'angolo visuale della ricerca - che lo scopo dell'indagine è, comunque, nell'aspirazione a definire, in termini incontrovertibili, i rapporti tra giurisdizione e processo.

Al di là delle singole, individuali, posizioni che, di volta in volta, attestano il tentativo di ritrovare univoci riscontri a supporto di un'idea di giurisdizione già maturata, due sono 
gli aspetti costantemente presenti: la necessarietà del processo e la sua funzione di garanzia.

Ciò che ha alimentato diversità di opinioni è stato, infatti, l'intendersi sul rapporto tra l'una e l'altra dimensione del fenomeno processuale.

La cura con la quale tanto la Costituzione, quanto le Carte internazionali dei diritti si preoccupano di assicurare che la verifica giudiziale dell'illiceità del fatto avvenga nel rispetto dei diritti inalienabili della persona (presunta non colpevole fino alla condanna definitiva) legittima l'interprete ad affermare, almeno nella dimensione costituzionale del processo, che non è questo il fine dell'accertamento giudiziale, certamente non deputato a realizzare il comando della legge sostanziale.

Il diritto processuale penale moderno, nato all'epoca dell'Illuminismo, con l'affermazione dell'esigenza della legalità nell'accertamento penale - garantita dall'indipendenza dei giudici dal potere esecutivo, dall'obbligatorietà dell'azione penale e dal giudice naturale precostituito per legge -, risentiva tuttavia già allora di un'evoluzione del diritto penale condizionato dalle differenti tradizioni giuridiche, dalle diverse impostazioni politico-costituzionali e dalle "emergenze criminali" del momento, le quali hanno profondamente inciso sull'interpretazione e l'applicazione dei criteri della legalità processuale.

Quando si parla di difesa sociale il tema centrale è quello delle misure cautelari che, consentendo ante iudicium ai giudici di privare della libertà personale le persone raggiunte da gravi indizi di colpevolezza, superano la presunzione di innocenza che, per l'art. 27 della Costituzione italiana, deve valere fino alla sentenza definitiva di condanna. Le esigenze della difesa sociale ripropongono, in sostanza, sul piano processuale, i problemi e i dilemmi che esse pongono sul piano sostanziale.

Su quest'ultimo, la difesa sociale implica una maggiore discrezionalità, e quindi un certo conflitto col principio di stretta legalità, proponendo, soprattutto, quel quesito sulla sentenza indeterminata, che difficilmente può ritenersi compatibile con il brocardo nulla poena sine lege.

Ma, non si può non ammettere, di contro, che la giustizia stessa, intesa anche come adeguazione in senso soggettivo, e cioè come vera giustizia, esige, indipendentemente da qualsiasi considerazione di difesa sociale, un'indagine della personalità del soggetto per adeguare la pena alla misura della colpa (nulla poena sine culpa).

Cosicché, anche le esigenze della giustizia non coincidono sempre con quelle della legalità, mentre possono coincidere con quelle della difesa sociale.

Ebbene, tutti questi valori sembrano, oggi, vuoti contenitori, mentre i contenuti sono rappresentati dalle nuove norme approvate in tema di misure di prevenzione, soprattutto in ambito personale.

Per dirla in modo forse eccessivamente semplicistico, stante la lentezza dell'accertamento giurisdizionale "ordinario" (forse per il suo eccessivo "garantismo"), il legislatore, chiamato a fronteggiare le continue emergenze di criminalità, sia di carattere organizzato che comune, ha deciso di ampliare le categorie di reati per le quali il semplice "sospetto" (quindi in mancanza di un completo e garantito accertamento giurisdizionale) è sufficiente per privare un soggetto della propria libertà, in nome di un crescente - e a volte frainteso - allarme criminale che giustifica il ricorso ad altre forme di difesa sociale. Le misure di prevenzione, sebbene sempre esistite nel nostro ordinamento - al pari delle misure di sicurezza e cautelari - sono state negli ultimi anni potenziate in ragione di un "riordino" avvenuto nell'ambito del Codice Antimafia. 
Il tutto secondo il pronunciamento del giudice penale che, sebbene in quest'ambito sia "legato" a criteri di giudizio fondati anche solo sul mero "sospetto", dà l'impressione (ma solo quella) che l'accertamento sia assolutamente garantito secondo quei valori (e quei principi) su esposti.

Ebbene, proprio per quest'aura di assoluta legalità, al legislatore è stato sufficiente ampliare la categoria di reati di "maggior allarme sociale" per consentire il ricorso alle misure di prevenzione - fortemente limitanti la libertà personale e reale dell'individuo non più soltanto per quelli di criminalità organizzata e terrorismo in genere, ma anche per quelli, ad esempio, di corruzione, di violenza sessuale e, da ultimo, di stalking, garantendo la difesa sociale, paradossalmente, proprio attraverso la giurisdizione penale.

E se tali misure assumono sempre più le caratteristiche di vera e propria pena applicata senza processo, ecco che allora l'insoddisfazione e la preoccupazione del garantista ne sono dirette conseguenze.

\section{La prevalente logica della prevenzione: una confusione terminologica}

La continua ricerca di equilibrio tra "autorità" e "libertà" nel contrasto delle emergenze sociali diviene via via ardua. Lo stress cui il sistema giustizia è stato sottoposto in questi ultimi anni - a causa di emergenze sociali quali criminalità organizzata, terrorismo e corruzione, nonché la complessità dei fattori e delle relazioni che tra essi intercorrono rende sempre più difficile elaborare una strategia di contrasto efficace.

A partire dal 2012, l'esperienza peninsulare ha fatto registrare uno spropositato aumento dei dispositivi di contrasto ai fenomeni corruttivi, tanto da modificare profondamente gli strumenti processuali di accertamento, con un evidente pericolo di erosione delle soglie di garanzia offerte a soggetti indagati e/o imputati di tali tipi di reati, con la conseguente creazione, invece, di potenziali situazioni in cui l'anticipazione eccessiva della soglia di punibilità di alcune ha portato il sistema penale ad una pericolosa deriva inquisitoria che non corrisponde alla logica e alla cultura di chi ha ispirato tale cambiamento.

L'inadeguatezza del tessuto normativo, incapace di reprimere le condotte penalmente rilevati, ha portato ad un aggiornamento dell'intero sistema di contrasto, aggiungendo dei dispositivi di prevenzione e di trasparenza dell'operato della pubblica amministrazione e intervenendo sulle norme sostanziali e processuali.

Nel sistema giuridico italiano il fenomeno della corruzione è stato affrontato in precedenza esclusivamente in chiave penalistica, mentre negli altri ordinamenti giuridici se ne è accolto un concetto più ampio: non solo i fatti penalmente rilevanti, ma ogni fenomeno di abuso e strumentalizzazione della funzione pubblica per conseguire vantaggi indebiti di natura personale. Alle misure repressive sanzionatorie sono state affiancate numerose misure di carattere preventivo, grazie alla L. 190/2012 (c.d. anticorruzione o anche "Legge Severino"). Ed è così che le tendenze internazionali in ambito preventivorepressivo sono entrate anche in Italia. È proprio il comma 1 dell'art. 1 della L. 190/2012 a stabilire che la stessa, in attuazione dell'art. 6 della Convenzione dell'Organizzazione delle Nazioni Unite contro la corruzione e degli articoli 20 e 21 della Convenzione penale sulla corruzione, "individua, in ambito nazionale, l'Autorità nazionale anticorruzione [ANAC] e gli altri organi incaricati di svolgere, con modalità tali da assicurare azione coordinata, attività di controllo, di prevenzione e di contrasto della corruzione e dell'illegalità nella pubblica amministrazione". La relazione illustrativa del disegno di legge ha da subito delineato l'obiettivo che si intendeva perseguire: prevenzione e 
repressione del fenomeno della corruzione attraverso un approccio multidisciplinare, nel quale gli strumenti sanzionatori si configurano solamente come alcuni dei fattori per la lotta alla corruzione e all'illegalità nell'azione amministrativa. Nello specifico, si pongono a sostegno del provvedimento legislativo motivazioni di trasparenza e controllo provenienti dai cittadini e di adeguamento dell'ordinamento italiano agli standard internazionali: la corruzione, infatti, danneggia la credibilità di un paese soprattutto da un punto di vista economico, poiché disincentiva gli investimenti, anche stranieri, frenando di conseguenza lo sviluppo.

Al potere giudiziario vengono affiancate, dunque, due nuove forze nella lotta alla corruzione amministrativa: le pubbliche amministrazioni e i suoi dipendenti e l'opinione pubblica. Con l'istituzione delle autorità di vigilanza, identificate nell'ANAC a livello centrale e nei responsabili della prevenzione della corruzione presso ogni P.A., viene a crearsi un network piramidale di sorveglianza e controllo, organizzato secondo i piani anticorruzione predisposti: attraverso una sorta di adeguamento del modello della L. 231/2001 alla Pubblica amministrazione, la prevenzione alla corruzione passa attraverso misure anticipatorie volte a rafforzare la legalità nella gestione di qualsiasi procedimento amministrativo, con l'individuazione di nuovi soggetti (le autorità di vigilanza) e la predisposizione di nuovi strumenti (i piani anticorruzione), sia a livello centrale che periferico.

Al tradizionale strumento penale-repressivo si affiancano, dunque, misure operanti soprattutto sul versante amministrativo e destinate a costituire una funzione preventiva.

Da un punto di vista prettamente processuale, la riforma della legge anticorruzione ha inciso sulla disciplina del cd. patteggiamento, poiché, al comma 1-ter, ne subordina l'accesso alla restituzione integrale del prezzo o del profitto del reato, nonché sulla confisca per l'equivalente ex art. 322-ter c.p. (estendendola anche al profitto), in quanto «nel caso di condanna, o di applicazione della pena su richiesta delle parti a norma dell'art. 444 c.p.p. per uno dei delitti previsti dagli artt. 314 a 320, anche se commessi da soggetti indicati dall'art. 322-bis, comma 1, è sempre ordinata la confisca dei beni che costituiscono "il prezzo, il prodotto o il profitto del reato". Tuttavia, disciplinando la confisca per l'equivalente, la seconda parte dell'articolo prevede che, quando non sia possibile procedere alla confisca di questi, essa si estenda sui beni che sono nella disponibilità del reo per un valore corrispondente al prezzo o al profitto (quest'ultimo riferimento è stato appunto inserito dalla L. 190/12).

Tale tecnica anticipatoria delle soglie di punibilità è dovuta anche alla percezione sociale del fenomeno in Italia. Nel 2018, 1'85\% dei cittadini, infatti, era convinto che i livelli di malaffare fossero alti, ma non ha vissuto episodi sulla propria pelle né su quella dei familiari. Questi sono i dati rilevati del Corruption Perceptions Index di Transparency International, le quali fotografano non l'integrità delle istituzioni in maniera oggettiva, ma la percezione soggettiva della corruzione. Ciononostante risultano essere un parametro importate per gli investitori, i quali "confluiscono in misura maggiore laddove sono assicurate certezza del diritto e istituzioni integre". I risvolti economici di tutto ciò, dunque, sono significativi. Tuttavia, dal 2012 ad oggi, l'Italia ha guadagnato +11 punti.

Certamente questo risulta essere un passo in avanti alla luce dell'attenzione dedicata al tema, ma tale risultato può ritenersi un successo alla luce dell'anticipazione delle soglie di tutela e della conseguente, pericolosa, deriva inquisitoria?

Seppure tali indici siano indiscutibilmente redatti seguendo meticolosi criteri di professionalità restano pur sempre dei giudici meramente valutativi, sganciati da 
esperienze dirette. Ed infatti, guardando ai dati che registrano le esperienze dirette della popolazione con il fenomeno corruttivo, la situazione cambia. Non v'è dubbio che la problematica resti, e che sia indiscutibilmente un problema sociale, ma il report dell'Istat svolto nel 2017, riporta che "il 7,9\% delle famiglie nel corso della vita sia stato coinvolto direttamente in eventi corruttivi quali richiesta di denaro, favori, regali o altro in cambio di servizi o agevolazioni”. Da ciò si può dedurre che gli episodi in qualche modo riconducibili alla corruzione, senza distinguerli per entità, sarebbero più o meno 18 mila in un anno e, dunque, probabilmente molto meno di quanto l'indice di percezione non faccia presumere.

La causa di tutto ciò, probabilmente, può ricondursi anche al peso della comunicazione politica e dei media sul tema negli ultimi anni, circostanza che ha deviato l'analisi dai dati obiettivi - e utilizzabili per un'ottimizzazione degli strumenti di contrasto - facendo sì che la discussione sul tema possa essere basata su valutazioni opinabili e da enfatizzazioni strumentali.

Ciò accade proprio a causa del paradosso degli indici percettivi e la loro fallacia - alla base, tuttavia, delle leggi in materia nel nostro Paese e, in particolar modo, della L. cd. "spazzacorrotti" (L. 3 del 2019) - secondo il quale "più si perseguono i fenomeni corruttivi sul piano della prevenzione e le fattispecie di reato sul piano della repressione, maggiore è la percezione del fenomeno".

Attraverso tali indici assistiamo, infatti, alla valorizzazione della percezione soggettiva, senza considerare in alcun modo le caratteristiche istituzionali e normative del Paese considerato.

La distorsione macroeconomica generata dal paradosso, dunque, è destinata a risultare un neo indelebile, un circolo vizioso in cui l'inasprimento delle misure repressive volte al contenimento del fenomeno e i metodi comunicativi adoperati, ne incrementano la percezione.

\section{Quale possibile bilancio, dunque, alla luce della disamina della normativa attualmente vigente?}

Una risposta potrebbe rinvenirsi nella comparazione: nulla di tutto l'imponente apparato del sistema italiano di prevenzione alla corruzione e tutela della trasparenza è paragonabile a quanto è avvenuto, invece, in Albania con l'approvazione unanime del Parlamento, della nota legge costituzionale di riforma della giustizia della Repubblica d'Albania (L. 76/2016), con cui ne sono stati ridisegnati profondamente gli assetti istituzionali.

La riforma, nata sotto la spinta degli Stati Uniti e dell'Unione europea con l'appoggio del Governo, ha risposto all'incalzante esigenza di limitare lo strapotere dei magistrati, sempre più impigliati nelle maglie della corruzione, sospettati di legami con il mondo del crimine e, soprattutto, di una personale amministrazione della cosa pubblica; è stata altresì diretta ad arginare l'enorme influenza del potere politico sulla magistratura, puntando ad aumentarne il livello di professionalità, al fine di restituire la fiducia dei cittadini nelle istituzioni.

La legge è in qualche misura simbolica, percepita come porta di accesso all'Unione europea. In questa prospettiva, è stata ridisegnata la struttura della Corte di Cassazione, della Corte Costituzionale, del Consiglio Superiore della Giustizia, ora Consiglio Superiore Giudiziario, così come è stato rivisto il ruolo del Presidente della Repubblica e 
le sue prerogative in materia di giustizia. Inoltre, sono stati istituiti dei nuovi organismi di rilevo costituzionale: il Consiglio Superiore della Procura, l'Alto Ispettore di Giustizia, la Procura Speciale e il Tribunale Speciale per gli high crimes nonché per i reati commessi da politici e alti funzionari dello Stato, il Consiglio per le Nomine nella Giustizia e in via transitoria due commissioni di primo e secondo grado, note come il "vetting", che dovranno esaminare tutte i dossier, le condotte professionali, personali e i patrimoni dei singoli magistrati attualmente in servizio.

Di particolare interesse è non solo il procedimento di valutazione dei magistrati attuali da parte della Commissione Indipendente e dal Collegio di Appello, ma la fase istruttoria che precederà la loro valutazione. Tale fase è caratterizzata da un procedimento tipicamente inquisitorio, rappresentato da uno step di indagini sui magistrati, specificamente riguardanti la valutazione della provenienza lecita del patrimonio, controllo della figura stessa del magistrato e delle relazioni familiari, la valutazione delle capacità professionali, relazionate per iscritto dinanzi alla Commissione e poi una ulteriore fase istruttoria che potrà condursi da parte degli stessi commissari della Commissione di rivalutazione.

Alla luce di questo quadro, deve darsi conto di alcune perplessità destate da tale sistema, specie riguardo al procedimento di valutazione: se, da un lato, è vero che sia la Costituzione sia la L. 86 del 2016 prevedono che le prove raccolte nell'ambito del procedimento del "vetting" non possano essere utilizzate contro giudici e pubblici ministeri nell'ambito di eventuali procedimenti penali contro costoro, dall'altro, le strutture deputate al "vetting" sono controllate dal Governo. Inoltre, la legge non presenta chiarezza in merito ai criteri che verranno utilizzati dai commissari della procedura, per la valutazione delle attività professionali dei giudici e pubblici ministeri.

Non può tuttavia biasimarsi la drasticità della riforma della giustizia in Albania, caratterizzata per anni da un sistema intriso di corruzione. Le criticità insite in questo meccanismo, così nuovo, ma già così problematico, non sono tardate ad arrivare.

Per superare queste criticità ed effettivamente indagare e perseguire i crimini commessi dagli alti funzionari è stata più di recente istituita in Albania la SPAK, la nuova procura speciale anti-corruzione e anti-criminalità organizzata. Questa, composta da due organi, ovvero la procura speciale, composta da procuratori, e l'agenzia nazionale per le indagini $(\mathrm{BKH})$, è un organo indipendente, non politicizzato, istituito proprio al fine di superare il vetting, ovvero il processo di rivalutazione previsto dalla riforma del sistema giudiziario. La forza stessa della SPAK è nella sua natura di istituzione costituzionale, che le offre la forza e l'indipendenza dalle possibili modifiche degli "intoccabili".

E sul piano internazionale, dove poi si gioca la vera partita, la collaborazione Italia Albania è oggi continua e proficua. Proprio nel luglio di quest'anno, sul tema della corruzione è stata avviata un'importante collaborazione tra ANAC, OSCE e ALSAI, la Corte dei Conti albanese. Lo scopo principale è raggiungere alti standard di scambio internazionale di dati e informazioni, così come buone pratiche ed esempi virtuosi. L'obiettivo a lungo termine è quello di individuare criteri oggettivi al fine di misurare la corruzione in un Paese, utilizzando dati in tempo reale. Solo analizzando indicatori oggettivi si potrà "misurare" il peso della corruzione su un Paese e, di conseguenza, avviare azioni più incisive per combatterla.

La questione, quindi, può dirsi tutt'altro che risolta, ma probabilmente si è oggi sulla giusta direzione. 


\section{Conclusioni}

L'esempio dell'Albania potrebbe rappresentare un punto focale per paesi che non hanno una radicale tradizione democratica e, dunque, non per l'Italia, il cui tessuto democratico è ormai consolidato.

Tuttavia, entrambe le esperienze, con le dovute proporzioni, palesano profonde criticità. Infatti, se l'Albania ha puntato decisamente tutto sulla cultura delle misure di prevenzione per la repressione (con innegabili effetti-domino che minano fortemente la stabilità democratica della giovane repubblica), l'Italia - seppur di più longeve tradizioni democratiche - ha invece optato per un'apparente soluzione preventiva, favorendo piuttosto un chiaro ritorno a logiche vetero-inquisitorie da applicare attraverso il procedimento penale, destinato a ricoprire nuovamente un ruolo centrale nelle dinamiche politiche, in ciò che sembra sempre più un ritorno al diritto penale teso a concretizzare la concettualizzazione del diritto penale del nemico, ossia quella "giustificativa", in alcuni casi, di un binario parallelo e separato del diritto penale.

Sul piano dei diritti, esibisce due livelli di garanzia diversa, perché si rivolge a due categorie differenti di soggetti: il primo vige per il cittadino ordinario, il secondo, invece, è uno strumento da poter utilizzare contro chi, di volta in volta, viene identificato come nemico all'interno di una data società.

Dal punto di vista processuale, è poi evidente che, almeno in Italia, il regime del "doppio binario", dapprima destinato solo ai reati di criminalità organizzata e terrorismo (quelli, cioè, per antonomasia a forte caratterizzazione di pericolosità sociale), sia, oggi, indubbiamente applicato anche ai processi per la repressione di reati contro la P.A. o, più in generale, ai fenomeni corruttivi, quasi a voler attribuire, a questi, l'etichetta di "reati di maggior allarme sociale", poiché capaci di sovvertire l'ordine democratico del paese.

Una risposta così determinata del legislatore, tuttavia, non tranquillizza, non soddisfa le esigenze di difesa sociale, né pare poter risolvere equamente il fenomeno della corruzione che, come noto, è primariamente culturale: nonostante si possa sostenere, da un punto di vista scientifico, che ciò dovrebbe suggerire (se non imporre) l'adozione di provvedimenti che lentamente semplifichino la pubblica amministrazione, assistiamo invece ad un processo di inesorabile ritorno a modelli di repressione che, in ragione della loro invasività, rischiano di perseguitare i cittadini e di non perseguire i crimini.

Con il conseguente - e inevitabile - aumento esponenziale di rischi correlati alla commissione di errori giudiziari, dovuto anche alla palese anticipazione della soglia di punibilità che, come ulteriore grave effetto, avrà quello di precludere a soggetti solo "sospettati" di tali tipi di reati di intraprendere - o dover interrompere - carriere politiche per il semplice "allarme" scaturito dalla condizione di essere soltanto indagati (dunque, nemmeno imputati).

Inteso come valutazione dell'illiceità di un fatto, un giudizio può venir formulato anche senza le vere ed effettive garanzie della giurisdizione: si tratterà, in tal caso, di un giudizio etico, politico o di altra natura; non sarà certamente un giudizio espresso secondo diritto dal giudice del processo penale: solo attraverso il modello accertativo di matrice giurisdizionale, il giudizio sul fatto può aver valore nel mondo degli effetti giuridici. 


\section{References}

(1) I "principi" esprimono le garanzie che debbono trovare riconoscimento nella disciplina del processo e, come tali, postulano l'attuazione dei loro contenuti. I "caratteri" costituiscono gli strumenti attraverso i quali quei "principi" si realizzano, rivelando la "fisionomia" del processo, il suo "volto", la tecnica a mezzo della quale esso si struttura.

(2) L'esperienza storica ci ha dimostrato che col metodo inquisitorio non si tendeva ad accertare alcunché, perché, immediatamente formulata, l'accusa era ritenuta affermazione già provata. Il disvalore del comportamento veniva colto prima ancora che si iniziasse ad inquisire il soggetto, sottoposto ad investigazione non già per ricavare dalla ricostruzione del fatto un'opinio delicti, ma per confortare, con conferme solenni e formali e a mezzo di meccanismi rituali, una riprovevolezza già asserita.

(3) Per un approfondimento sul concetto di giurisdizione, si rinvia a A.A. DALIA, La garanzia della giurisdizione penale, in Annali dell'Istituto di Diritto e Procedura Penale, 1992.

(4) Secondo l'ancora condivisibile pensiero di P. NUVOLONE, Legalità, giustizia e difesa sociale, atti della Conferenza del 5 agosto 1964, presso la Facoltà di diritto dell'Università di Panarà.

(5) Infatti, leggendo l'attuale formulazione dell'art. 4 del Codice antimafia, è lunghissimo l'elenco dei reati che consentono l'applicazione di misure di prevenzione personale, tra l'altro anche per i soggetti indiziati del delitto di cui all'articolo 640-bis o del delitto di cui all'articolo 416 del Codice penale, finalizzato alla commissione di alcuni dei più gravi delitti contro la P.A.

(6) Per intenderci, attraverso il ricorso alle misure di prevenzione - misure di carattere amministrativo e di pubblica sicurezza con una veste di giurisdizionalità - si è voluto non altro che dare una risposta concreta ed immediata al "problema" della sicurezza che, se poteva essere giustificata per le "classiche" categorie di reati per cui il ricorso a misure "straordinarie" poteva trovare la sua ratio nella natura stessa di questi crimini "straordinari", tuttavia non tranquillizza gli operatori che sono nati nella cultura della garanzia della giurisdizione e che si vedono oggi traditi da quanto sta avvenendo nel nostro Paese.

(7) Secondo R. CANTONE, Il sistema della prevenzione della corruzione in Italia, in penalecontemporaneo.it, 27 novembre 2017, solo un sistema basato sulla valorizzazione della trasparenza dell'operato della P.A., congiuntamente ad un'adeguata riforma del codice penale e processuale penale, può contribuire ad un'efficace prevenzione dei fenomeni corruttivi.

(8) Del resto, lo stesso R. CANTONE, 'Percezione' della corruzione e politiche anticorruzione in penalecontemporaneo.it, 18 febbraio 2019, ha affermato che "con questo impianto penale 1'Italia ha affrontato la manifestazione più grave di corruzione mai emersa nella storia del paese, quella degli anni 90 del precedente secolo, divenuta nota come Tangentopoli o Mani pulite che riguardò il sistema dei partiti e della politica, entrambi travolti dal ciclone giudiziario, tanto che, secondo gli storici, l'esito delle indagini e dei processi cagionò un tale cambiamento da giustificare il passaggio alla seconda Repubblica. Il sistema delineato, però, era apparso non adeguato già rispetto a quelle forme corruttive di Tangentopoli e a maggior ragione è risultato non a passo con i tempi rispetto a quelle che via via si sono delineate successivamente, quelle cioè che vedono sempre più corrotti e corruttori partecipi indistinti di vere e proprie associazioni a delinquere, finalizzate a saccheggiare le risorse pubbliche".

(9) La volontà del legislatore è stata quella di ridare vigore ai principi di cui agli artt. 28, 54 e 97 Cost, i quali fanno riferimento, oltre che ai diritti e doveri dei cittadini, anche alla P.A.

(10) La Legge 6 novembre 2012, n. 190, Disposizioni in materia di prevenzione e repressione della corruzione e dell'illegalità nella pubblica amministrazione, in Gazzetta Ufficiale n.265, del 13 novembre 2012, prevede nuovi obblighi e adempimenti per le amministrazioni pubbliche, attraverso modifiche al codice penale, deleghe legislative e rinvii ad atti secondari da emanare.

(11) In estrema sintesi, sono tre i momenti (i pilastri, potremmo con un po' di enfasi dire) che caratterizzano la strategia della prevenzione, sia pure in una prospettiva unitaria che resta quella della "corruzione", da contenere ed evitare. Il primo pilastro della strategia è connesso ad un capovolgimento della prospettiva tradizionale. L'esigenza di assicurare la legalità e la corretta cura degli interessi pubblici. Il secondo pilastro di questa strategia è nel diverso rapporto che deve intercorrere fra amministrazione e cittadini; sono questi ultimi i "beneficiari" dell'attività dell'amministrazione; i funzionari pubblici, sia quelli elettivi che quelli burocratici, gestiscono il potere nell'interesse della collettività e, in ultima analisi, quindi dei cittadini. A costoro devono dar conto - è il dar conto che viene espresso in un felice vocabolo, della tradizione anglosassone, "accountability" - e sono costoro che possono (e devono) chiedere ragione dell'operato dell'amministrazione. Un terzo gruppo di misure rivolge la sua attenzione alla figura del funzionario pubblico, ai suoi doveri e ai suoi comportamenti. L'idea di fondo perseguita è di rafforzare l'imparzialità 
"soggettiva" dell'amministrazione, e cioè di evitare situazioni di conflitto di interessi che rappresentino un rischio concreto di fatti corruttivi, di favorire l'emersione, anche attraverso la trasparenza, di eventuali interessi privati che possono pregiudicare la migliore cura dell'interesse pubblico e regolare le condotte individuali dei funzionari, così R. CANTONE, Il contrasto alla corruzione. Il modello italiano, in penalecontemporaneo.it, 2 ottobre 2018.

(12) Infatti, al fine di realizzare un'azione di prevenzione integrata tra i diversi soggetti che svolgono funzioni e attività amministrative, anche strumentali, i modelli di organizzazione e di gestione degli enti pubblici economici e degli enti di diritto privato in controllo pubblico di cui all'art. 6 del D.lgs. 231/2001 considerano anche solo il rischio di fenomeni corruttivi, individuando al loro interno le aree a maggior pericolo di corruzione, incluse quelle previste nell'art. 1, co. 16, della L. 190 del 2012, valutate in relazione al contesto, all'attività e alle funzioni dell'ente, prevedono la programmazione della formazione dei piani triennali anticorruzione, con particolare attenzione alla previsione di procedure per l'attuazione delle decisioni dell'ente in relazione al rischio di fenomeni corruttivi. Ai sensi dell'art. 1, co. 9, lett. f), nell'ambito del piano di prevenzione sono infatti individuati specifici obblighi di trasparenza, rendendosi necessario un raccordo tra il Piano per la prevenzione alla corruzione e il Piano per la trasparenza, rectius, tra il Responsabile per la prevenzione della corruzione e il Responsabile per la trasparenza.

(13) Comportando per questa ragione numerosi problemi ermeneutici a causa della successione di leggi penali nel tempo. Per una disamina delle citate questioni, si consenta il rinvio a G. DALIA, Le linee di demarcazione tra la nuova fattispecie di concussione e quella di induzione indebita a dare o promettere utilità (Cass., SS.UU., 14 marzo 2014, n. 12228). Il "caso Ruby" (Corte d'Appello di Milano, Sez. II, 16 ottobre 2014), in M. SANTISE, F. ZUNICA (a cura di), Coordinate ermeneutiche di diritto penale, Torino, 2015, pp. 97-107. La L. 190/2012 ha avuto, chiaramente, anche un effetto sulla disciplina sostanziale, modificando le vecchie fattispecie, inasprendo il trattamento sanzionatorio, e coniandone di nuove. Gli effetti più evidenti di questo cambio di rotta si sono manifestati con l'innalzamento delle pene previste per le fattispecie di peculato, corruzione propria, corruzione in atti giudiziari e abuso d'ufficio. Per quanto attiene al delitto di concussione, invece, è stato scisso in due nelle figure di concussione ex art. 317 c.p. e di induzione indebita a dare o promettere utilità ex art. 319-quater c.p., limitando la prima alla sola condotta di costrizione. Contestualmente, e contrariamente a quanto stabilito con la 1 86/1990 (che aveva esteso la punibilità per tale reato anche all'incaricato di pubblico servizio), nel 2012 rispondeva per il reato p. e p. ex art. 317 solo il pubblico ufficiale. Tale scelta fu così tormentata che, con la L. 69/2015, il legislatore ha ripristinato la precedente struttura. La legge, altresì, ha distinto la corruzione propria, relativa al compimento di un atto contrario ai doveri di ufficio, dalla corruzione impropria, rimodulando quindi la figura delittuosa, punendo - come già detto - la corruzione tra privati (ex art. 2635 c.c.) e introducendo, con l'art 346-bis c.p., la nuova fattispecie delittuosa del traffico di influenze illecite.

(14) Così il già Presidente dell'ANAC, ossia l'Autorità italiana anticorruzione, R. CANTONE, Il contrasto alla corruzione. Il modello italiano, cit.

(15) T. PADOVANI, La spazzacorrotti, riforma delle illusioni e illusioni della riforma, in Archivio penale, Fasc. 3, 2018, pp. 1-11.

(16) Così G. TARTAGLIA POLCINI, Il paradosso di Trocadero, in Il diritto penale della Globalizzazione, 22 ottobre 2017.

(17) Così E. BOZHEKU, La Riforma costituzionale ("strutturale”) del sistema della giustizia nella Repubblica d'Albania, in penalecontemporaneo.it, 16 ottobre 2016, per il quale si tratta di "un procedimento di natura squisitamente inquisitoria (di comunista memoria; verrebbe da dire alla faccia del processo accusatorio propugnato dagli americani nella loro veste di "nobili" portatori di democrazia e giustizia nel mondo!) dove è prevista una istruttoria formale da parte di una serie di soggetti che dovranno svolgere delle vere e proprie indagini sui magistrati, per poi relazionare per iscritto dinanzi alla Commissione e poi una ulteriore fase istruttoria che potrà condursi da parte degli stessi commissari della Commissione di rivalutazione".

(18) Così Giuseppe Busia, Presidente dell'ANAC.

(19) Cfr. S. KEIL, Z. ARKAN, The EU and Member State Building. European Foreign Policy in the Western Balkans, Londra-New York, 2015.

(20) Elaborata dal noto penalista Günther Jakobs, presentata per la prima volta, in via indiretta, nel 1985, nella Relazione alle giornate dei Penalisti tedeschi a Francoforte sul Meno, per poi essere ripresa con più convinzione, dal medesimo autore, nella Relazione al Congresso celebrato a Berlino nel 1999 su "la Scienza del Diritto penale alle soglie del nuovo millennio". Tale elaborazione teorica ha acquisito negli ultimi tempi nuova importanza nel contesto della lotta al terrorismo, nel solco del diritto penale "emergenziale". 
